\title{
Targeting the overexpressed CREB inhibits esophageal squamous cell carcinoma cell growth
}

\author{
PING CHEN, MIAOMIAO LI, QIANYUN HAO, XUESONG ZHAO and TAO HU \\ College of Basic Medical Sciences, Zhengzhou University, \\ Collaborative Innovation Center of Henan Province for Cancer Chemoprevention, \\ Zhengzhou, Henan 450001, P.R. China
}

Received July 17, 2017; Accepted December 13, 2017

DOI: $10.3892 /$ or.2017.6167

\begin{abstract}
Although several studies highlight the important role of cAMP-responsive element binding protein (CREB) in tumor progression, little is known concerning the expression and function of CREB in esophageal cancer. In the present study, the expression of CREB was evaluated using a human esophageal squamous cell carcinoma tissue array by immunohistochemical analysis, which was confirmed by western blot analysis of tissues from esophageal cancer, and adjacent esophageal tissue. The role of CREB on esophageal cancer cell growth was analyzed in vitro and in vivo. Results showed that CREB was overexpressed in esophageal squamous cell carcinomas tissues, which was positively correlated with lymph node metastasis and tumor-node-metastasis (TNM) stage of esophageal cancer patients. Downregulating the expression of CREB effectively reduced esophageal cell growth in vitro and in vivo, induced $\mathrm{S}$ phase cell cycle arrest, triggered apoptosis and inhibited cell migration and invasion. These findings suggested CREB as an attractive drug target for esophageal cancer.
\end{abstract}

\section{Introduction}

Esophageal cancer is a refractory disease and the sixth leading cause of cancer-related deaths worldwide $(1,2)$. Esophageal squamous cell carcinoma (ESCC) is the major histologic subtype in Asia (1,2). Lack of effective diagnosis and prognosis marker account for the patients diagnosed at late stage and poor prognosis (3). It is important to identify new molecular target and develop novel therapeutic regiment for esophageal cancer.

Transcription factor has now been explored as attractive anticancer target (4). Previous studies showed that

Correspondence to: Dr Tao Hu, College of Basic Medical Sciences, Zhengzhou University, Collaborative Innovation Center of Henan Province for Cancer Chemoprevention, Zhengzhou, Henan 450001, P.R. China

E-mail: hnhutao@zzu.edu.cn

Key words: esophageal cancer, CREB, cell growth, cell migration
cAMP-responsive element binding protein (CREB) play an important role in tumor progression. For example, CREB was overexpressed in non-small cell lung cancer (NSCLC) and significantly associated with decreased survival duration in never smokers with NSCLC (5). CREB promoted abnormal proliferation and survival of myeloid cells in vitro and in vivo (6). Overexpressed CREB was detected in acute myeloid leukemia (6), acute lymphoblastic leukemia (7) and associated with relapse disease or a lower overall survival. Downregulation of CREB inhibited cancer cell growth (8-10), migration and invasion (11-14). However, Liu et al reported that the expression of CREB from Juvenile myelomonocytic leukemia patients was significantly lower than that from normal adults (15). Targeting CREB promoted cell proliferation in Hodgkin lymphoma (16). These results implied that CREB play an important role in tumor progression in a tumorspecific manner. However, the expression and role of CREB in ESCC remains elusive.

Here we found that CREB was overexpressed in esophageal squamous cell carcinoma tissues, which was positively associated with lymph node metastasis and tumor-node-metastasis (TNM) stage of ESCC patients. Knockdown of CREB reduced cell growth in vitro and in vivo, induced $\mathrm{S}$ phase arrest, triggered apoptosis, inhibited cell migration and invasion. These results imply that CREB may be an attractive anticancer target in ESCC.

\section{Materials and methods}

Cell lines. The human esophageal squamous cell carcinoma cell lines (EC1, EC9706, EC109, TE1, TE13, Kyse140 and Kyse450) were grown in Dulbecco's modified Eagle's medium (HyClone, Logan, UT, USA) supplemented with 10\% FBS [Biological Industries (BI), Inc., Cromwell, CT, USA] at $37^{\circ} \mathrm{C}$ with $5 \% \mathrm{CO}_{2}$. Human immortalized normal esophageal epithelial cell line (Het-1A) was a kind gift from Professor R. Liu (Southeast China University) and was cultured in Bronchial Epithelial Cell Medium (BEGM; BulleKit).

Immunohistochemistry (IHC) staining of human esophageal cancer tissue array. Human esophageal squamous cell carcinoma tissue array was purchased from Xi'an Alenabio Biotech Co. Ltd. (Xi'an, China). IHC staining was carried 
out with specific CREB antibody (Abcam Trading Co. Ltd., Shanghai, China). Briefly, the tissue array sections $(5 \mu \mathrm{m})$ were dehydrated and peroxidase blocked. Primary antibodies were added and incubated at $4^{\circ} \mathrm{C}$ overnight, followed by staining with a Histostain-SAP kit (ZSGB-BIO, Beijing, China). The slides were counterstained with hematoxylin. The stained slides were observed by microscopy, and images were acquired. Based on staining intensity, samples were classified into five groups from the lowest density (-) to the highest (++++) as previously described $(17,18)$.

Collection of esophageal cancer tissues. Esophageal cancer and adjacent esophageal tissues were collected from esophageal squamous cell carcinoma patients undergoing resection at the Linzhou Cancer Hospital (Linzhou, Henan, China) from July 2012 to September 2014. Histological diagnosis and tumor-node-metastasis (TNM) stages of cancers were determined in accordance with the American Joint Committee on Cancer (AJCC) manual criteria for esophageal cancer.

Gene silencing using small interfering RNA (siRNA). Knockdown of CREB was carried out using siRNA oligonucleotides. The sequences of the siRNA are as follows: siCREB-1, CCAAGUUGUUGUUCAAGCU; siCREB-2, GAGAGAGGU CCGUCUAAUG; siControl, UUCUCCGAACGUGUCACGU.

Western blotting. Kyse 450 and EC1 cells were transfected with siControl or siCREB, and then proteins were collected for western blot analysis, using antibodies against CREB (Abcam Trading Co. Ltd.), P27, WEE1, cleaved caspase-3, cleaved poly(ADP)-ribose polymerase (PARP) (Cell Signaling, Boston, MA, USA). CDC2, CDK2, cyclin B, cyclin A1, cyclin D, cyclin E (Abgent Biotech Co., Ltd., Suzhou, China). Tubulin (Shanghai Likun Trade Co. Ltd., Shanghai, China) was used as a control. Densitometric analysis for the quantification relative to tubulin was performed using the ImageJ software.

Cell viability and clonogenic assay. The cell viability was determined by Cell Counting Kit-8 (CCK-8; Beyotime Biotech Co., Haimen, China). EC1 and Kyse 450 cells were transfected with siControl or siCREB for $24 \mathrm{~h}$, and then seeded into 96 -well plates $\left(2.5 \times 10^{3}\right.$ cells/well) for 72 or $96 \mathrm{~h}$. Subsequently, CCK-8 solution was added to each well and incubated at $37^{\circ} \mathrm{C}$ for $2 \mathrm{~h}$. The absorbance at $450 \mathrm{~nm}$ was measured.

For the clonogenic assay, cells were transfected with siControl or siCREB, seeded into 6-well plates with 500 cells/well in triplicate, and then incubated for 12 days. The colonies were fixed by $4 \%$ paraformaldehyde, stained using crystal violet solution, and then the colonies with $>50$ cells were counted.

Cell cycle analysis. EC1 and Kyse 450 cells were transfected with siControl or siCREB for $72 \mathrm{~h}$, and then cells were harvested, fixed in $70 \%$ ethanol at $-20^{\circ} \mathrm{C}$, stained with propidium iodide (PI; $50 \mu \mathrm{g} / \mathrm{ml})$ containing RNase A (30 $\mu \mathrm{g} / \mathrm{ml})$ (both from Sigma, St. Louis, MO, USA) at $37^{\circ} \mathrm{C}$ for $30 \mathrm{~min}$, and analyzed for cell cycle profile by flow cytometry (FACScan; Becton-Dickinson, Franklin Lakes, NJ, USA). Data were analyzed using ModFit LT software (Verity Software House, Inc., Topsham, ME, USA). The expression of cell cycle-related proteins was detected using indicated antibodies.
Apoptosis assay and detection of the caspase- 3 activity. Cells were transfected with siControl or siCREB for $96 \mathrm{~h}$. Apoptosis was determined using the Annexin V-FITC/PI apoptosis kit (BioVision, Inc., Mountain View, CA, USA) according to the manufacturer's instructions. The activities of caspase-3 were measured using the CaspGLOW assay kit (BioVision, Inc.) according to the manufacturer's instructions. Cell proteins after transfection were collected, and then cleaved PARP and caspase-3 were detected.

Invasion assay. The invasion assay was carried out in Matrigel (Becton-Dickinson)-coated Transwell inserts with a pore size of $8-\mu \mathrm{m}$, as previously described (17). Briefly, the inserts were pre-coated with Matrigel. EC1 cells $\left(3 \times 10^{4}\right)$ transfected with siRNA for $48 \mathrm{~h}$ were seeded in serum-free medium in the upper chamber, whereas medium with $10 \%$ FBS was added to the lower well. After incubating for $24 \mathrm{~h}$, the cells in the upper chamber were carefully removed with a cotton swab. The inserts were fixed in methanol, stained with $0.4 \%$ crystal violet, observed under microscopy, and images were acquired. Then, the dye was eluted by $33 \%$ acetic acid and detected at OD $570 \mathrm{~nm}$.

Wound healing assay. For wound-healing assay, cells were seeded on 6-well plates and the confluent monolayer was scratched by a plastic pipette, then cells were washed three times with PBS. Images were captured at 0 or $36 \mathrm{~h}$ after wounding.

In vivo assay. The stable $\mathrm{EC} 1$ cell line with lentivirus targeting CREB was established as previously described $(19,20)$. BALB/c nude female mice were subcutaneously injected with $5 \times 10^{6}$ EC1 cells stably expressing lenti-shCREB or lenti-shControl, respectively. Tumor growth was observed and tumor area was recorded twice a week with a FluorVivo Model-300 imaging system (INDEC BioSystems, Santa Clara, CA, USA) (19). At the time of sacrifice, tumor tissues were harvested, photographed and weighed. Animal experiments were performed in accordance with animal protocols approved by the Institutional Animal Care and Use Committee of Zhengzhou University.

Statistical analysis. The statistical significance of differences between groups was assessed using GraphPad Prism 5 software (GraphPad Software Inc., La Jolla, CA, USA). The t-test was used for the comparison of parameters between groups. For all tests, two levels of significance $\left({ }^{*} \mathrm{P}<0.05 ;{ }^{* * *} \mathrm{P}<0.01\right)$ were applied.

\section{Results}

CREB is overexpressed in esophageal squamous cell carcinoma tissues. To investigate whether CREB served as anti-esophageal cancer target, we firstly examined the expression levels of CREB by immunochemistry (IHC) staining of the tissue array derived from human ESCC. Results showed that CREB was overexpressed in ESCC tissues (Fig. 1A), which was confirmed by western blot assay using the esophageal cancer and adjacent esophageal tissues from esophageal squamous cell carcinoma patients (Fig. 1B). Furthermore, the overexpressed CREB was positively correlated with lymph 
Table I. Correlation between the expression of CREB and clinical characteristics of ESCC patients.

\begin{tabular}{|c|c|c|c|c|}
\hline \multirow[b]{2}{*}{ Characteristics } & \multirow[b]{2}{*}{ Total $\mathrm{n}$} & \multicolumn{2}{|c|}{ CREB no. } & \multirow[b]{2}{*}{ P-value } \\
\hline & & Negative $\mathrm{n}(\%)$ & Positive n (\%) & \\
\hline Overall & 114 & $18(16)$ & $96(84)$ & - \\
\hline Sex & & & & 0.804 \\
\hline Male & 85 & $13(15)$ & $72(85)$ & \\
\hline Female & 29 & $5(17)$ & $24(83)$ & \\
\hline Age (years) & & & & 0.725 \\
\hline$<60$ & 55 & $8(15)$ & $47(85)$ & \\
\hline$\geq 60$ & 59 & $10(17)$ & $49(83)$ & \\
\hline Lymph node metastasis $(\mathrm{N})$ & & & & 0.029 \\
\hline No & 38 & $10(26)$ & $28(74)$ & \\
\hline $\mathrm{N} 1-2$ & 76 & $8(11)$ & $68(89)$ & \\
\hline TNM stage & & & & 0.018 \\
\hline I & 14 & $6(43)$ & $8(57)$ & \\
\hline II & 62 & $6(10)$ & $56(90)$ & \\
\hline III & 35 & $6(17)$ & $29(83)$ & \\
\hline IV & 3 & $0(0)$ & $3(100)$ & \\
\hline Tumor invasion & & & & 0.849 \\
\hline $\mathrm{T} 1$ & 14 & $2(14)$ & $12(86)$ & \\
\hline $\mathrm{T} 2$ & 50 & $9(18)$ & $41(82)$ & \\
\hline $\mathrm{T} 3$ & 50 & $7(14)$ & $43(86)$ & \\
\hline Differentiation & & & & 0.577 \\
\hline Well & 21 & $3(14)$ & $18(86)$ & \\
\hline Moderate & 49 & $7(14)$ & $42(86)$ & \\
\hline Poor & 44 & $8(18)$ & $36(82)$ & \\
\hline
\end{tabular}

${ }^{\mathrm{a}} \mathrm{P}<0.05$ was defined as significant, Fisher's exact test. CREB, cAMP-responsive element binding protein; ESCC, esophageal squamous cell carcinoma; TNM, tumor-node-metastasis.

node metastasis and TNM stage of ESCC patients (Table I). However, CREB was expressed higher in ESCC cell lines than human immortalized normal esophageal epithelial cell line (Het-1A) (Fig. 1C). These results implied that CREB may be an attractive anti-ESCC target.

Targeting CREB inhibits cell growth of ESCC. According to the above results, we next examined the effect of knockdown CREB on cell growth. Results showed that the expression of CREB was effectively downregulated using specific siRNA (Fig. 2A). Silencing of CREB inhibited cell growth by cell proliferation (Fig. 2B) and colony formation assay (Fig. 2C).

Knockdown of CREB induces $S$ cell cycle arrest in esophageal cancer cells. To elucidate the growth suppression mechanism by CREB silencing, the cell cycle profile was examined after knockdown of CREB. As shown in Fig. 3, knockdown of CREB induced $\mathrm{S}$ cell cycle arrest (Fig. 3A) and downregulated the expression of cyclin A1 and D (Fig. 3B).

Silencing of CREB triggers apoptosis in esophageal cancer cells. We next examined whether apoptosis was also responsible for the growth inhibition effect of CREB silencing. Results showed that knockdown of CREB-induced apoptosis, as evident by increased Annexin V-positive cells (Fig. 4A), improved caspase-3 activity (Fig. 4B) and enhanced expression level of cleaved caspase-3 and cleaved PARP (Fig. 4C).

Targeting CREB inhibits cell invasion and metastasis of ESCC. Statistical analysis results showed that the overexpressed CREB was correlated with lymph node metastasis (Table I). So the expression of CREB was downregulated by siRNA and the effect on cell invasion and metastasis was examined. Results showed that silencing CREB inhibited ESCC cell invasion and metastasis by Transwell (Fig. 5A) and wound healing assay (Fig. 5B).

Targeting CREB inhibits ESCC cell growth in vivo. To further investigated the growth suppressive effect of CREB knockdown in vivo, BALB/c nude female mice were subcutaneously injected with $5 \times 10^{6} \mathrm{EC} 1$ cells stably expressing lenti-shCREB (marked as shCREB) or lenti-shControl (marked as shControl), respectively. Tumor growth was observed and tumor area was recorded twice a week. Results showed that CREB silencing 
A

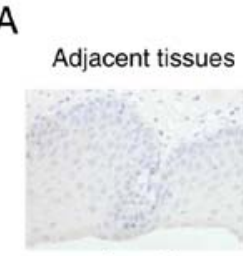

$(n=10)$

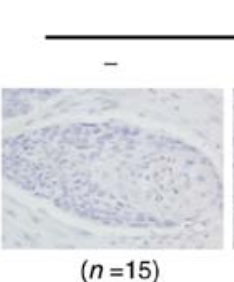

$(n=15)$
Tumor tissues

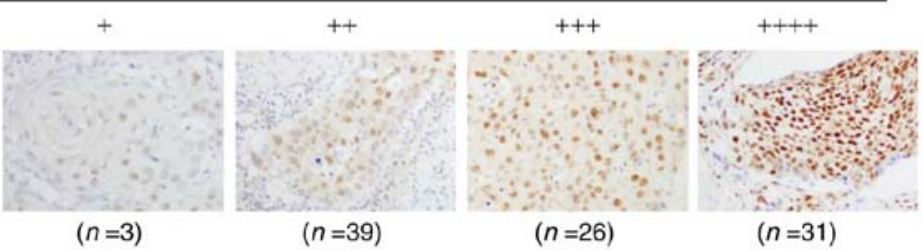

$(n=39)$

C
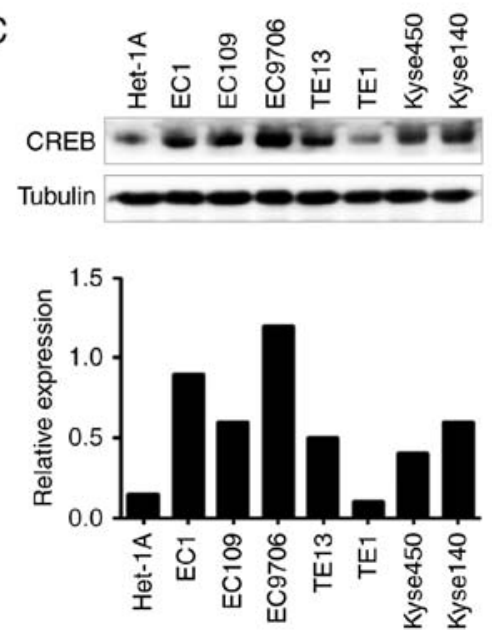

Figure 1. CREB is overexpressed in esophageal squamous cell carcinoma tissues. (A) The expression of CREB in human esophageal squamous cell carcinoma tissues was detected by immunohistochemistry (IHC) staining. According to staining intensity, samples were classified into five groups with increasing staining intensity from the weakest (-) to the strongest (++++). Representative images are shown. The number of each group is marked below the image. (B) Western blot analysis to determine the expression of CREB in ESCC and adjacent esophageal tissues. Representative results are shown in the upper panel and analysis of western blotting is shown in the lower panel. A, adjacent tissues, T, tumor tissues. (C) Expression of CREB in esophageal squamous cell carcinoma cell lines and human immortalized normal esophageal epithelial cell line (Het-1A). Results of western blotting are shown in the upper panel and analysis is shown in the lower panel.

\section{A}
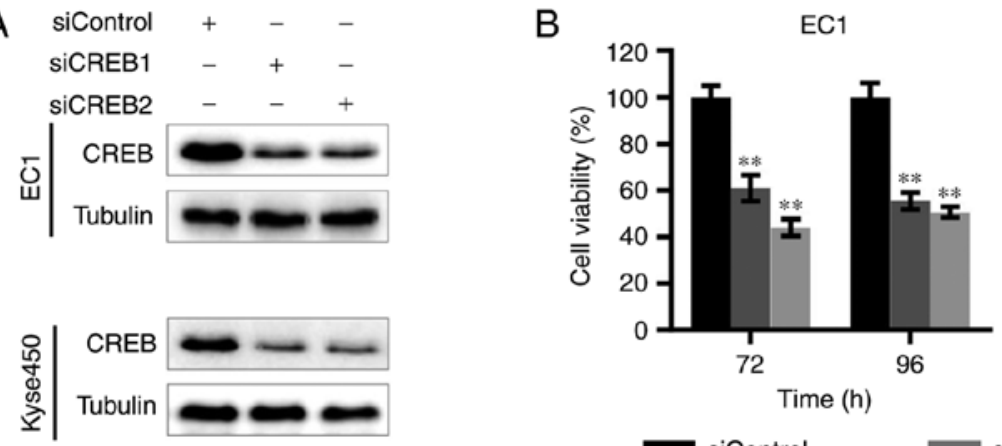

siControl

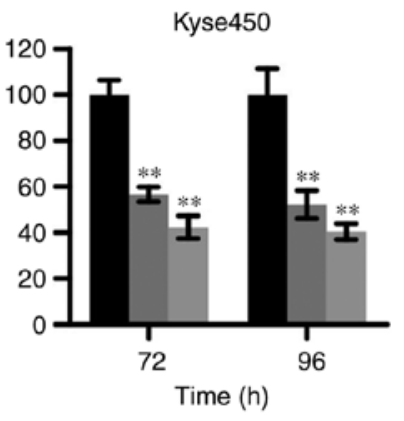

SICREB1

Kyse450

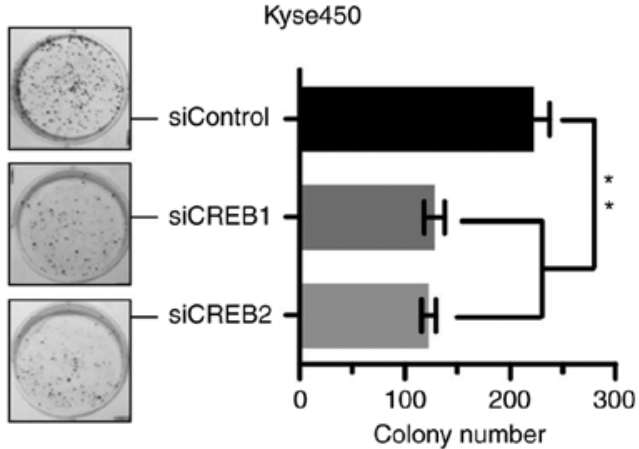

Figure 2. Knockdown of CREB inhibits cell proliferation of human esophageal cancer. (A) Detection of siRNA efficiency targeting CREB. Cells were transfected with siRNA for $96 \mathrm{~h}$, and then proteins were collected and knockdown efficiency was determined by western blotting. (B) Effect of silencing CREB on the viability of esophageal squamous cell carcinoma cells EC1 and Kyse450. Cells were transfected with siRNA for 72 or 96 h and viability was assessed using the CCK-8 assay. (C) Effect of silencing CREB on clonogenic survival of esophageal squamous cell carcinoma cells EC1 and Kyse450. Representative images are shown in the left panel and colony count in the right panel. The statistical significance of differences between groups was assessed according to Materials and methods $\left(\right.$ P $\left.<0.05 ;{ }^{* *} \mathrm{P}<0.01\right)$. 
A
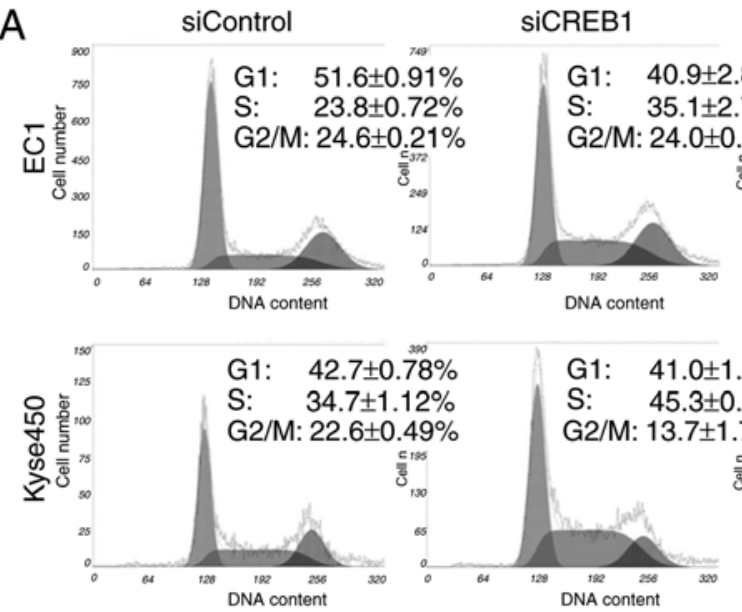

G1: $\quad 41.0 \pm 1.46 \%$ S: $\quad 45.3 \pm 0.75 \%$ $\begin{array}{lr}\text { S: } & 45.3 \pm 0.75 \% \\ \text { G2/M: } 13.7 \pm 1.75 \%\end{array}$

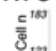

$5_{123}^{180}$ SiCREB2

G1: $\quad 39.4 \pm 1.54 \%$ S: $\quad 32.7 \pm 1.99 \%$ G2/M: $27.9 \pm 0.58 \%$

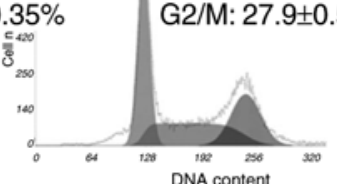

G1: $\quad 41.1 \pm 0.36 \%$ S: $\quad 43.0 \pm 0.72 \%$ G2/M: $15.9 \pm 0.40 \%$

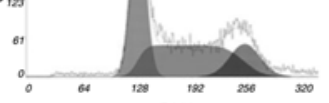

$\mathrm{B}$

\begin{tabular}{|c|c|c|}
\hline & EC1 & Kyse450 \\
\hline Control & + & + \\
\hline siCREB1 & - & - \\
\hline ICREB2 & + & - \\
\hline $\mathrm{CDC} 2$ & $--1=$ & $-2-1$ \\
\hline CDK2 & -- & -- \\
\hline Cyclin A1 & 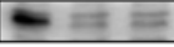 & $-\cdots$ \\
\hline Cyclin B & $-\infty$ & $-\infty$ \\
\hline Cyclin D & $E-m$ & $--\cdots$ \\
\hline Cyclin E & -- & -2 \\
\hline P27 & $-\infty$ & $-1-$ \\
\hline WEE1 & -2 & --- \\
\hline Tubulin & $-\infty$ & - \\
\hline
\end{tabular}

siControl $\square$ siCREB1 siCREB2

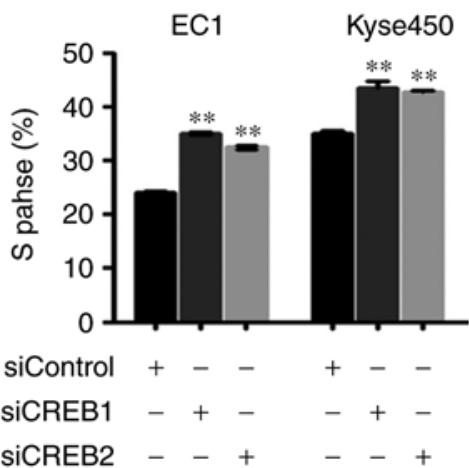

Figure 3. Knockdown of CREB induces S cell cycle arrest of human esophageal cancer cells. (A) EC1 and Kyse450 cells were transfected with siRNA, and then stained with PI staining. DNA contents were analyzed by fluorescence-activated cell sorting (FACS) analysis. Representative images are shown (left panel). The statistical significance of differences between groups was assessed using the GraphPad Prism 5 software $\left.{ }^{* *} \mathrm{P}<0.01\right)$ were applied (right panel). (B) Effect of silencing CREB on the expression of cell cycle-related proteins was detected using indicated antibodies.

suppressed tumor growth over time while control tumors grew rapidly, as revealed by real-time images of tumors (Fig. 6A), tumor growth curve (Fig. 6B; $\mathrm{P}<0.05)$, tumor size (Fig. 6C) and tumor weight analysis (Fig. 6D; $\mathrm{P}<0.05$ ).

\section{Discussion}

Despite the improvement in the surgical and non-surgical therapy for ESCC (2), the general outcome remains very poor for overall 5-year survival rates $(\sim 10 \%)$ and 5-year post-esophagectomy survival rates $(\sim 15-40 \%)$ (21). Diagnosis at advanced stage and resistance to chemotherapy still affect the refractory disease. Therefore, it is urgent to find new therapeutic targets.

CREB belongs to basic/leucine zipper (bZIP) transcription factor family (22) and is described as a proto-oncogene $(6,23)$. CREB play an important role at early stage of papilloma formation (24), promoted abnormal proliferation of myeloid cells in vitro and in vivo and was implicated in myeloid cell transformation (6). However, CREB was overexpressed in ovarian adenocarcinoma (10), non-small cell lung $(9,25)$ and breast cancer (12), leukemia (26), and highly associated with disease stage or poor clinical outcomes of the patients. In different conditions, CREB participated in tumorigenesis and influenced melanoma (14,27), T cell and myeloid leukemia (6) and hepatocellular carcinoma (28). Overexpressed CREB promoted tumor progression by regulating cell proliferation, cell cycle, apoptosis, angiogenesis or metastasis. These findings highlight a pivotal role of CREB in carcinogenesis. However, the expression and role of CREB in ESCC remains to be elucidated. In the present study, we reported that CREB was overexpressed in esophageal squamous cell carcinomas tissues, which was positively correlated with lymph node metastasis and TNM stage of ESCC patients. Moreover, knockdown of CREB significantly inhibited cell proliferation of ESCC cells in vitro and in vivo. These results indicated that CREB may be involved in ESCC cell growth.

Previous studies suggested CREB as a promising target for cancer therapy. Downregulating the expression of CREB by ectopic expression of dominant repressor CREB or siRNA against CREB suppressed the growth and survival of NSCLC cells and induced apoptotic cell death (9). Ectopic expression of dominant-repressor CREB inhibited acute myeloid leukemia cell proliferation in vitro and in vivo $(6,22)$, sensitized melanoma 
A

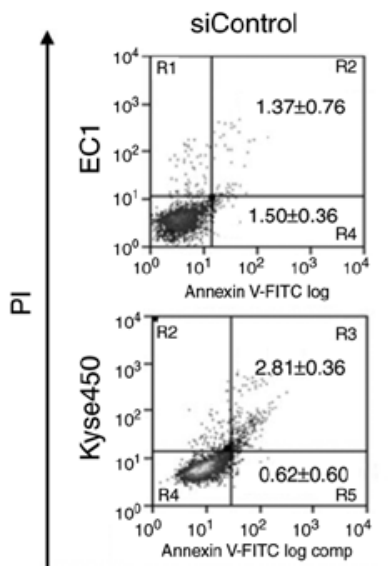

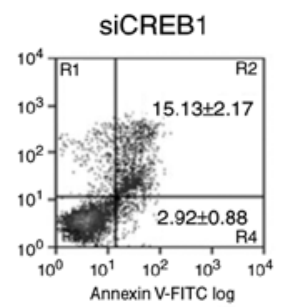
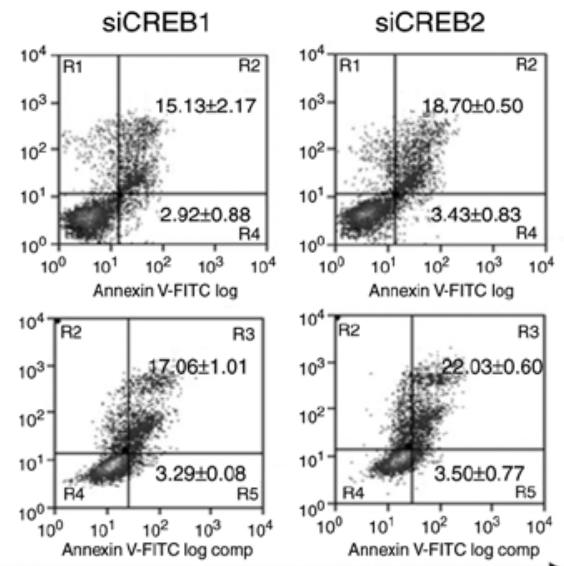

Annexin V-FITC

B
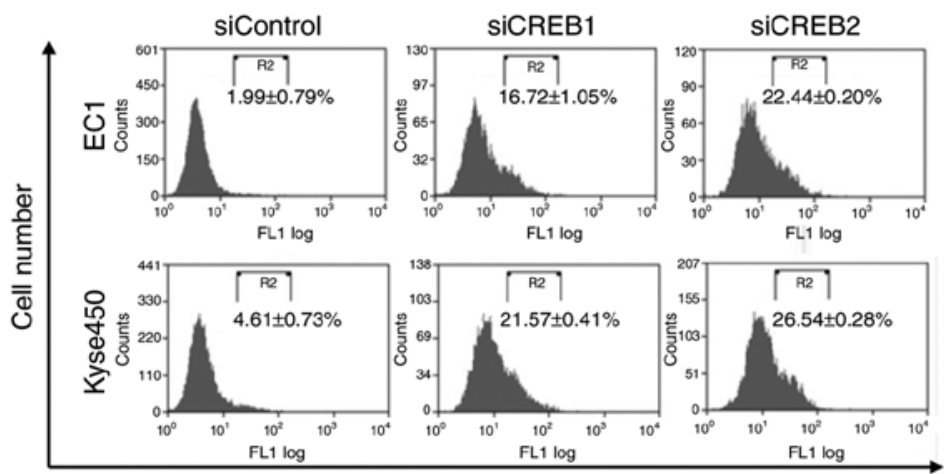

FITC

C

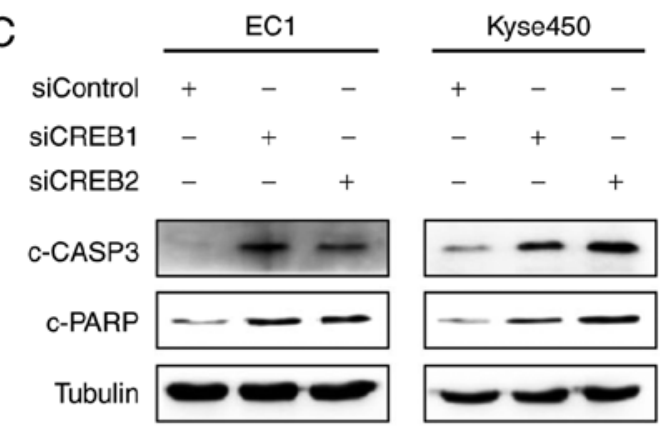

Figure 4. Knockdown of CREB triggers apoptosis of human esophageal cancer cells. EC1 and Kyse 450 cells were transfected with siRNA for $96 \mathrm{~h}$. Apoptosis was determined by Annexin V-FITC/PI double-staining analysis (A) Caspase-3 activity was analyzed by FACS (B) Cleaved PARP and caspase-3 were detected by western blot analysis (C). In the panel A and B, representative images are shown (left panel). The statistical significance of differences between groups was assessed using the GraphPad Prism5 software ( $\left.{ }^{* *} \mathrm{P}<0.01\right)$ (right panel).
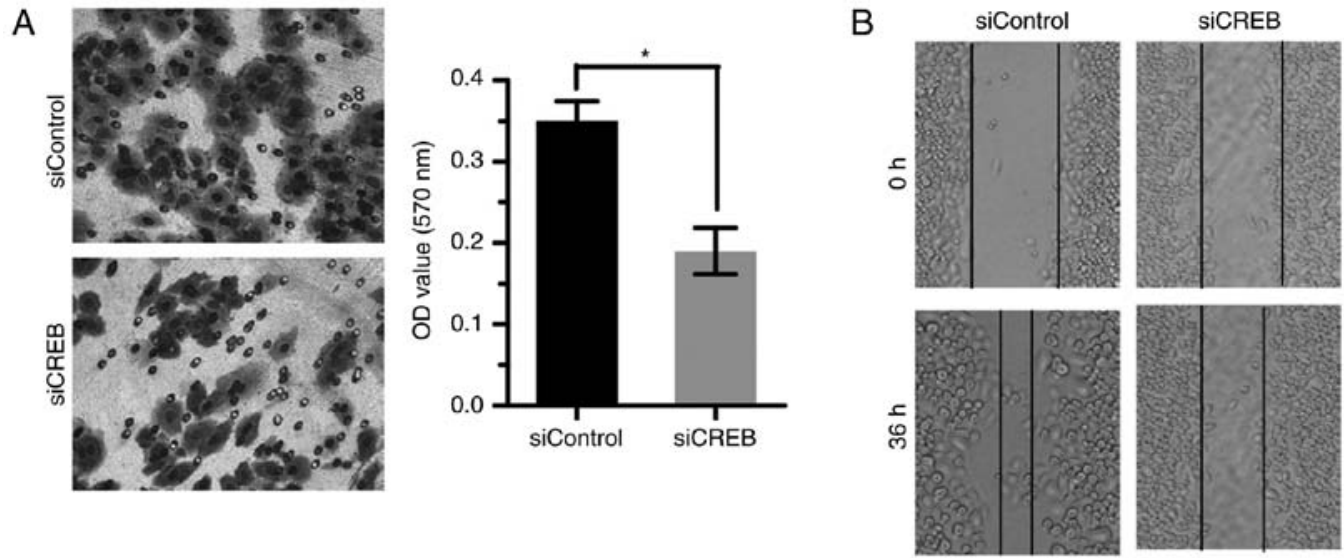

Figure 5. Knockdown of CREB inhibites cell migration and invasion in human esophageal cancer cells. EC1 cells were transfected with siControl or mixture of siCREB1 and siCREB2 for $48 \mathrm{~h}$. (A) Cell invasion was determined by Transwell assay. (B) Cell migration was detected by wound healing assay. 
A
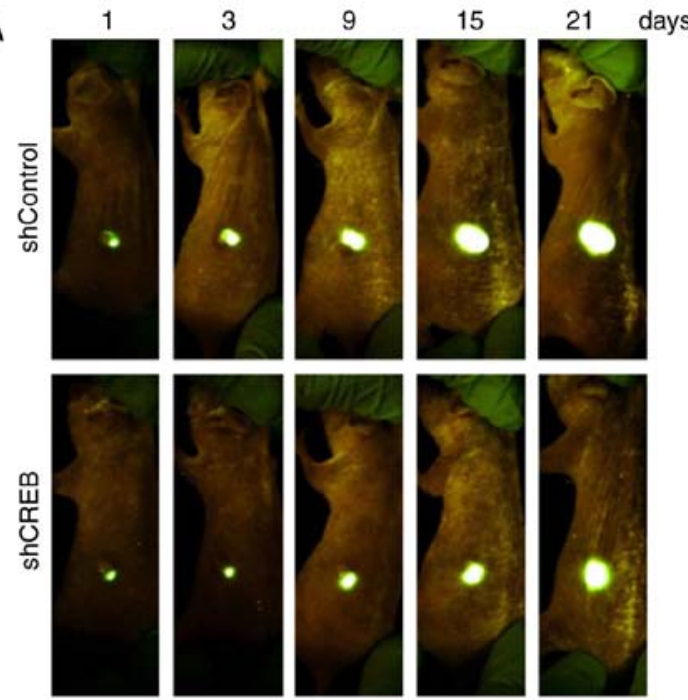

C

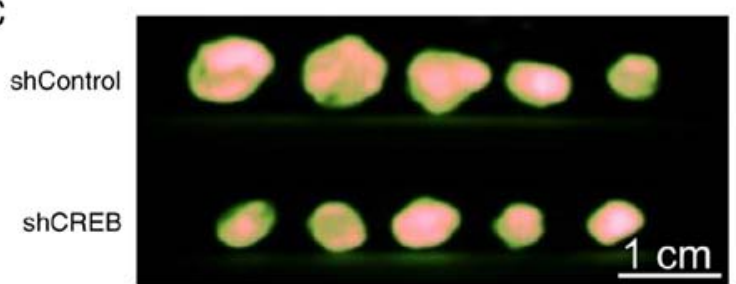

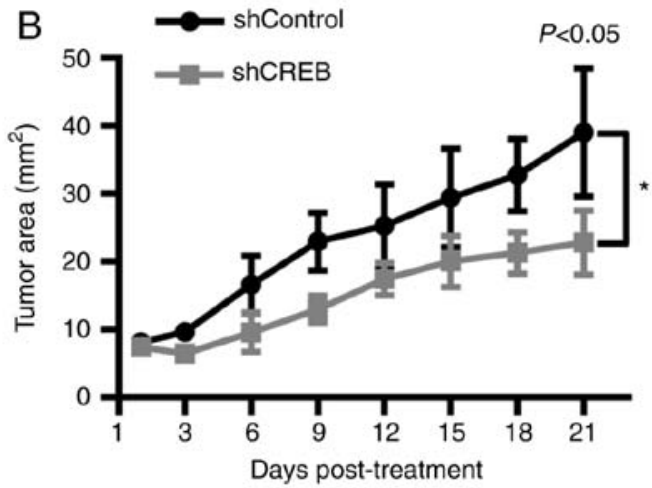

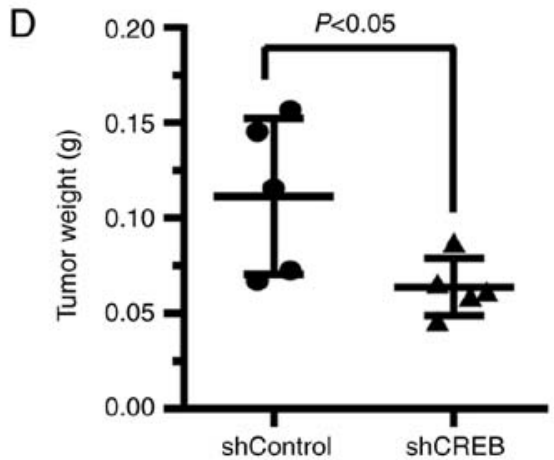

Figure 6. Knockdown of CREB suppresses esophageal tumor growth in vivo. Whole-body images of tumor model were captured (A). The data were converted to tumor growth curves (B). Mice were sacrificed and tumor tissues were harvested, photographed (C), and weighed (D) at the end of the study.

cells to apoptosis and downregulated their tumorigenicity and metastatic potential in nude mice $(14,27,29)$. CREB knockdown inhibited human pre-B acute lymphoblastic leukemia cell growth and induced cell apoptosis (8). Furthermore, several small compounds were reported to target CREB or inhibit its transcriptional activity, exhibited efficient anticancer effect and showed little to no toxicity to normal epithelial cells, fibroblasts or hematopoietic cells (30-34); 666-15, a CREB inhibitor, showed promising potency against breast cancer in vitro and in a mouse model. Moreover, the mice treated with 666-15 showed no evidence of changes in body weight, complete blood count, blood chemistry profile, cardiac contractility and tissue histology from liver, kidney and heart (34). In contrast, CREB was involved in cisplatin/gemcitabine resistance (35) or radio sensitivity $(36,37)$. These results implied potential of CREB-target therapy. Here, our results showed that CREB silencing suppressed esophageal tumor cell growth in a mouse model. Whether knockdown of CREB effectively sensitized esophageal cells to chemoherapy/radiotherapy still need to be further investigated.

Mechanistically, CREB was reported to be involved in the regulation of cell cycle machinery, including cyclin A1 and D1 $(22,28,38-40)$. Desdouets et al reported that CREB was involved in regulation the expression of cyclin A, a pivotal regulatory protein which was involved in the $S$ phase of the cell cycle $(40,41)$. However, Linnerth et al reported that knockdown CREB using siRNA significantly reduced ovarian tumor cell proliferation, while there was no effect on apoptosis in these cells (10). Lu et al indicated that downregulation of CREB promoted cell proliferation by mediating
G1/S phase transition in Hodgkin lymphoma (16). Inhibitor of the CREB signaling pathway Ro-31-8220 inhibited CREB activation and arrested the cell cycle at the G2-M phase (9). Here we found that knockdown of CREB downregulated the expression of cyclin A1 and D, induced S phase cell cycle arrest and apoptosis. These results implied that the anticancer mechanism of targeting CREB was in a tumor-specific manner.

Invasion and metastasis are the important characteristics of malignant tumor (42). Previous reports showed that CREB promoted cancer metastasis $(12,14,43,44)$. CREB regulated vascular endothelial growth factor expression and was involved in human prostate cancer bone metastasis (45). In melanoma, CREB mediated tumorigenesis and metastatic potential $(45,46)$. Downregulated CREB using a dominantnegative form of CREB and CREB silencing inhibited cell growth and metastasis (45). In accordance with these results, here we found that CREB was overexpressed in esophageal squamous cell carcinomas tissues, positively correlating with lymph node metastasis. Moreover, knockdown of CREB inhibited cell migration and invasion using wound healing and Transwell assay.

In summary, this is the first study to investigate the expression and clinicopathological significance of CREB in ESCC. Results demonstrated that CREB was hyperexpressed in human ESCC tissues and positively correlated with lymph node metastasis and TNM stage of esophageal cancer patients. In addition, knockdown of CREB effectively inhibited cell growth in vitro and in vivo. These findings expanded our knowledge of CREB in ESCC progression 
and suggested CREB as a novel drug target for esophageal cancer.

\section{Acknowledgements}

The present study was supported by the National Natural Science Foundation Grant of China (grant nos. 81001102, 81101894 and 81672421), the Natural Science Foundation of Henan Province (grant no. 162300410302), the Outstanding Young Talent Research Fund of Zhengzhou University (grant nos. 51999223 and 32210449), and the Student's Platform for Innovation and Entrepreneurship Training Program of Zhengzhou University (grant 258 no. 1210459106). The authors thank Professor Ran Liu from Southeast China University for kindly providing the Het-1A cell lines.

\section{References}

1. Torre LA, Bray F, Siegel RL, Ferlay J, Lortet-Tieulent J and Jemal A: Global cancer statistics, 2012. CA Cancer J Clin 65: 87-108, 2015.

2. Sohda M and Kuwano H: Current status and future prospects for esophageal cancer treatment. Ann Thorac Cardiovasc Surg 23: 1-11, 2017.

3. Belkhiri A and El-Rifai W: Advances in targeted therapies and new promising targets in esophageal cancer. Oncotarget 6: 1348-1358, 2015.

4. Darnell JE Jr: Transcription factors as targets for cancer therapy. Nat Rev Cancer 2: 740-749, 2002.

5. Seo HS, Liu DD, Bekele BN, Kim MK, Pisters K, Lippman SM, Wistuba II and Koo JS: Cyclic AMP response element-binding protein overexpression: A feature associated with negative prognosis in never smokers with non-small cell lung cancer. Cancer Res 68: 6065-6073, 2008.

6. Shankar DB, Cheng JC, Kinjo K, Federman N, Moore TB, Gill A, Rao NP, Landaw EM and Sakamoto KM: The role of CREB as a proto-oncogene in hematopoiesis and in acute myeloid leukemia. Cancer Cell 7: 351-362, 2005.

7. van der Sligte NE, Kampen KR, ter Elst A, Scherpen FJ, Meeuwsen-de Boer TG, Guryev V, van Leeuwen FN, Kornblau SM and de Bont ES: Essential role for cyclic-AMP responsive element binding protein 1 (CREB) in the survival of acute lymphoblastic leukemia. Oncotarget 6: 14970-14981, 2015.

8. Shabestari RM, Safa M, Alikarami F, Banan M and Kazemi A: CREB knockdown inhibits growth and induces apoptosis in human pre-B acute lymphoblastic leukemia cells through inhibition of prosurvival signals. Biomed Pharmacother 87: 274-279, 2017.

9. Aggarwal S, Kim SW, Ryu SH, Chung WC and Koo JS: Growth suppression of lung cancer cells by targeting cyclic AMP response element-binding protein. Cancer Res 68: 981-988, 2008.

10. Linnerth NM, Greenaway JB, Petrik JJ and Moorehead RA: cAMP response element-binding protein is expressed at high levels in human ovarian adenocarcinoma and regulates ovarian tumor cell proliferation. Int J Gynecol Cancer 18: 1248-1257, 2008.

11. Tan X, Wang S, Yang B, Zhu L, Yin B, Chao T, Zhao J, Yuan J, Qiang B and Peng X: The CREB-miR-9 negative feedback minicircuitry coordinates the migration and proliferation of glioma cells. PLoS One 7: e49570, 2012.

12. Son J, Lee JH, Kim HN, Ha H and Lee ZH: cAMP-responseelement-binding protein positively regulates breast cancer metastasis and subsequent bone destruction. Biochem Biophys Res Commun 398: 309-314, 2010.

13. Shukla A, Bosenberg MW, MacPherson MB, Butnor KJ, Heintz NH, Pass HI, Carbone M, Testa JR and Mossman BT: Activated cAMP response element binding protein is overexpressed in human mesotheliomas and inhibits apoptosis. Am J Pathol 175: 2197-2206, 2009.

14. Jean D and Bar-Eli M: Regulation of tumor growth and metastasis of human melanoma by the CREB transcription factor family. Mol Cell Biochem 212: 19-28, 2000.
15. Liu YL, Lensing SY, Yan Y, Cooper TM, Loh ML and Emanuel PD: Deficiency of CREB and over expression of miR-183 in juvenile myelomonocytic leukemia. Leukemia 27: 1585-1588, 2013

16. Huang GL, Liao D, Chen H, Lu Y, Chen L, Li H, Li B, Liu W, Ye C, Li T, et al: The protein level and transcription activity of activating transcription factor 1 is regulated by prolyl isomerase Pin1 in nasopharyngeal carcinoma progression. Cell Death Dis 7: e2571, 2016.

17. Li L, Wang M, Yu G, Chen P, Li H, Wei D, Zhu J, Xie L, Jia H, Shi J, et al: Overactivated neddylation pathway as a therapeutic target in lung cancer. J Natl Cancer Inst 106: dju083, 2014.

18. Chen P, Hu T, Liang Y, Li P, Chen X, Zhang J, Ma Y, Hao Q, Wang J, Zhang $\mathrm{P}$, et al: Neddylation inhibition activates the extrinsic apoptosis pathway through ATF4-CHOP-DR5 axis in human esophageal cancer cells. Clin Cancer Res 22: 4145-4157, 2016.

19. Hu T, Qi H, Li P, Zhao G, Ma Y, Hao Q, Gao C, Zhang Y, Wang C, Yang M, et al: Comparison of GFP-expressing imageable mouse models of human esophageal squamous cell carcinoma established in various anatomical sites. Anticancer Res 35: 4655-4663, 2015.

20. Hu T, Fu Q, Chen P, Zhang K and Guo D: Generation of a stable mammalian cell line for simultaneous expression of multiple genes by using $2 \mathrm{~A}$ peptide-based lentiviral vector. Biotechnol Lett 31: 353-359, 2009.

21. Huang FL and Yu SJ: Esophageal cancer: Risk factors, genetic association, and treatment. Asian J Surg: S1015-S9584, 2016.

22. Sakamoto KM and Frank DA: CREB in the pathophysiology of cancer: Implications for targeting transcription factors for cancer therapy. Clin Cancer Res 15: 2583-2587, 2009.

23. Steven A and Seliger B: Control of CREB expression in tumors: From molecular mechanisms and signal transduction pathways to therapeutic target. Oncotarget 7: 35454-35465, 2016.

24. Rozenberg J, Rishi V, Orosz A, Moitra J, Glick A and Vinson C: Inhibition of CREB function in mouse epidermis reduces papilloma formation. Mol Cancer Res 7: 654-664, 2009.

25. Liu W, Wu Y, Wang L, Gao L, Wang Y, Liu X, Zhang K, Song J, Wang H, Bayer TA, et al: Protein signature for non-small cell lung cancer prognosis. Am J Cancer Res 4: 256-269, 2014.

26. Pigazzi M, Ricotti E, Germano G, Faggian D, Aricò $M$ and Basso G: cAMP response element binding protein (CREB) overexpression CREB has been described as critical for leukemia progression. Haematologica 92: 1435-1437, 2007.

27. Jean D, Harbison M, McConkey DJ, Ronai Z and Bar-Eli M: CREB and its associated proteins act as survival factors for human melanoma cells. J Biol Chem 273: 24884-24890, 1998.

28. Abramovitch R, Tavor E, Jacob-Hirsch J, Zeira E, Amariglio N, Pappo O, Rechavi G, Galun E and Honigman A: A pivotal role of cyclic AMP-responsive element binding protein in tumor progression. Cancer Res 64: 1338-1346, 2004.

29. Leslie MC and Bar-Eli M: Regulation of gene expression in melanoma: New approaches for treatment. J Cell Biochem 94: 25-38, 2005.

30. Xie F, Li BX, Kassenbrock A, Xue C, Wang X, Qian DZ, Sears RC and Xiao X: Identification of a potent inhibitor of CREB-mediated gene transcription with efficacious in vivo anticancer activity. J Med Chem 58: 5075-5087, 2015.

31. Xie F, Li BX, Broussard C and Xiao X: Identification, synthesis and evaluation of substituted benzofurazans as inhibitors of CREB-mediated gene transcription. Bioorg Med Chem Lett 23: 5371-5375, 2013.

32. Mitton B, Hsu K, Dutta R, Tiu BC, Cox N, McLure KG, Chae HD, Smith M, Eklund EA, Solow-Cordero DE and Sakamoto KM: Small molecule screen for inhibitors of expression from canonical CREB response element-containing promoters. Oncotarget 7: 8653-8662, 2016

33. Li BX, Yamanaka K and Xiao X: Structure-activity relationship studies of naphthol AS-E and its derivatives as anticancer agents by inhibiting CREB-mediated gene transcription. Bioorg Med Chem 20: 6811-6820, 2012.

34. Li BX, Gardner R, Xue C, Qian DZ, Xie F, Thomas G, Kazmierczak SC, Habecker BA and Xiao X: Systemic inhibition of CREB is well-tolerated in vivo. Sci Rep 6: 34513, 2016.

35. Pan J, Li X, Wu W, Xue M, Hou H, Zhai W and Chen W: Long non-coding RNA UCA1 promotes cisplatin/gemcitabine resistance through CREB modulating miR-196a-5p in bladder cancer cells. Cancer Lett 382: 64-76, 2016. 
36. Zhang Y, Yu J, Liu H, Ma W, Yan L, Wang J and Li G: Novel epigenetic CREB-miR-630 signaling axis regulates radiosensitivity in colorectal cancer. PLoS One 10: e0133870, 2015.

37. Suarez CD, Deng $X$ and Hu CD: Targeting CREB inhibits radiation-induced neuroendocrine differentiation and increases radiation-induced cell death in prostate cancer cells. Am J Cancer Res 4: 850-861, 2014.

38. Pigazzi M, Manara E, Baron E and Basso G: miR-34b targets cyclic AMP-responsive element binding protein in acute myeloid leukemia. Cancer Res 69: 2471-2478, 2009.

39. De Falco V, Tamburrino A, Ventre S, Castellone MD, Malek M, Manié SN and Santoro M: CD44 proteolysis increases CREB phosphorylation and sustains proliferation of thyroid cancer cells. Cancer Res 72: 1449-1458, 2012.

40. Desdouets C, Matesic G, Molina CA, Foulkes NS, SassoneCorsi P, Brechot $\mathrm{C}$ and Sobczak-Thepot J: Cell cycle regulation of cyclin A gene expression by the cyclic AMP-responsive transcription factors CREB and CREM. Mol Cell Biol 15: 3301-3309, 1995.

41. Yoshizumi M, Wang H, Hsieh CM, Sibinga NE, Perrella MA and Lee ME: Down-regulation of the cyclin A promoter by transforming growth factor-beta1 is associated with a reduction in phosphorylated activating transcription factor-1 and cyclic AMP-responsive element-binding protein. J Biol Chem 272: 22259-22264, 1997.
42. Hanahan D and Weinberg RA: Hallmarks of cancer: The next generation. Cell 144: 646-674, 2011.

43. Dobroff AS, Wang H, Melnikova VO, Villares GJ, Zigler M, Huang L and Bar-Eli M: Silencing cAMP-response elementbinding protein (CREB) identifies CYR61 as a tumor suppressor gene in melanoma. J Biol Chem 284: 26194-26206, 2009.

44. Wang X, Ren Y, Zhuang H, Meng X, Huang S, Li Y, Hehir M and Wang P: Decrease of phosphorylated proto-oncogene $C R E B$ at Ser 133 site inhibits growth and metastatic activity of renal cell cancer. Expert Opin Ther Targets 19: 985-995, 2015.

45. Wu D, Zhau HE, Huang WC, Iqbal S, Habib FK, Sartor O, Cvitanovic L, Marshall FF, Xu Z and Chung LW: cAMP-responsive element-binding protein regulates vascular endothelial growth factor expression: Implication in human prostate cancer bone metastasis. Oncogene 26: 5070-5077, 2007.

46. Melnikova VO, Dobroff AS, Zigler M, Villares GJ, Braeuer RR, Wang H, Huang L and Bar-Eli M: CREB inhibits AP-2alpha expression to regulate the malignant phenotype of melanoma. PLoS One 5: e12452, 2010. 